\title{
Polarized exciton magnetoluminescence under resonance excitation of GaAs/AlAs type II superlattices
}

\author{
G.E. PIKUS and F.G. PIKUS* \\ A.F. Ioffe Physico-Technical Institute, 194021 St. Petersburg, Russia \\ * University of Utah, Salt Lake City, Utah 84112, U.S.A.
}

\begin{abstract}
The theory for the exciton magnetoluminescence in GaAs/AlAs superlattices is presented. We find the energies of the exciton states, taking into account an additional exchange splitting, caused by the symmetry reduction from $\mathrm{D}_{2 \mathrm{~d}}$ to $\mathrm{C}_{2 \mathrm{v}}$. We show that measurements of the luminescence polarization allow to choose between the alternative models of this splitting.
\end{abstract}

In type II GaAs/AlAs superlattices with $z$ axis parallel to [001], the holes are localised in the GaAs layers, and the electrons mainly in $X$ valleys of AlAs layers. It has been found experimentally (see [1-6] and references there) that in such structures there exist an additional exchange splitting of the heavy hole exciton state $\Gamma_{5}$, which is the state active in the luminescence. The split states $x^{\prime}$ and $y^{\prime}$ have their dipole moments parallel to [110] and [1 $\overline{1} 0]$, respectively. This splitting is attributed to the symmetry reduction, and should lead to the polarized luminescence, if the excitation is polarized along [100] or [010] axes. Instead, a non-polarized luminescence was observed ${ }^{1}$. The explanations proposed ${ }^{2-5}$ base on existence of the two kinds of the excitons, with splitting of the same magnitude $|2 \delta|$ but different sign. Moreover, the amount of excitons of both kinds is about the same. In Refs. [2, 3], this splitting is explained by the presence of local deformations $\varepsilon_{x y}$, which reduce the crystal symmetry from $D_{2 d}$ to $C_{2 v}$ and lead to the mixing of the heavy hole (hh) $| \pm 3 / 2\rangle$ and light hole (lh) $| \pm 1 / 2\rangle$ states. In another model ${ }^{4,5}$ the similar hh-lh mixing was attributed to the localisation of an exciton near one boundary between the well and the barrier. Then this mixing can be described by the appropriate boundary condition ${ }^{4}$, which takes into account that the symmetry of the ideal heterojunction $\mathrm{C}_{2 \mathrm{v}}$ is lower than the symmetry of the superlattice $\mathrm{D}_{2 \mathrm{~d}}$ :

$\varphi_{j}^{A}=\varphi_{j}^{B}, \quad \nabla_{z}^{j} \varphi_{j}^{A}-\nabla_{z}^{j} \varphi_{j}^{B}=\frac{1}{\sqrt{3}} t_{l-h} \sum_{j^{\prime}}\left(J_{x} J_{y}+J_{y} J_{x}\right) \varphi_{i j^{\prime}}^{B}$,

where $t_{l-h}$ is a real coefficient, $\varphi_{j}^{A, B}$ is the value of the hh wave function at the boundary, on the side of layer $A$ (GaAs) or $B$ (AlAs), and

$\nabla_{z}^{ \pm 3 / 2}=a_{0} \frac{m_{0}}{m_{h h}} \frac{\partial}{\partial z}, \quad \nabla_{z}^{ \pm 1 / 2}=a_{0} \frac{m_{0}}{m_{l h}} \frac{\partial}{\partial z}$.

Here $m_{0}$ is the free electron mass, $m_{h h}$ and $m_{l h}$ are the effective masses of hh and $l h$, respectively, and $a_{0}$ 
is the lattice constant.

In both cases, the exchange hamiltonian for the symmetry $\mathrm{C}_{2 \mathrm{v}}$ has the form:

$H_{e x}=\frac{2}{3}\left\{\Delta_{0}(\vec{J} \vec{\sigma})+\Delta_{1} \sum_{i} J_{i}^{3} \sigma_{i}+\delta\left(V_{x} \sigma_{y}-V_{y} \sigma_{x}\right)\right\}$,

where

$V_{i}=\left[J_{i}\left(J_{i+1}^{2}-J_{i+2}^{2}\right)\right]_{s i m m}, \quad i=x, y, z$,

with cyclic permutations of indexes, and

$[A B]_{\text {simm }}=1 / 2(A B+B A)$.

$J_{i}$ are the angular momentum operators in the basis of the hole eigenfunctions $| \pm 3 / 2\rangle,| \pm 1 / 2\rangle, \sigma_{i}$ are the Pauli matrices for electrons, and $\Delta_{0}, \Delta_{1}$, and $\delta$ are exchange interaction coefficients.

The optical properties of the exciton in the absence of magnetic filed do not depend upon the microscopic mechanism of the hh-lh mixing. As a result of the splitting of the exciton state with $J_{\mathrm{z}}= \pm 1$, the luminescence under the excitation, polarized along [100] or [010] (or under circularly polarized excitation), is non-polarized. However, as we show here, the constants in the hamiltonian $H_{\mathrm{B}}$, which describes the splitting of the excitonic states in a magnetic field, and, therefore, the resulting splitting, are very different in both models. This allows one to judge in favour of one of them.

The magnetic field induced splitting is described by the hamiltonian

$H_{\mathrm{B}}=\mu_{\mathrm{B}}\left\{\frac{1}{2} g_{e}\left(\sigma_{x} B_{x}+\sigma_{y} B_{y}\right)+g_{0}\left[q_{1}\left(J_{x}^{3} B_{x}+J_{y}^{3} B_{y}\right)+q_{2}\left(J_{x}^{3} B_{y}+J_{y}^{3} B_{x}\right)\right]\right\}$.

where $\mu_{\mathrm{B}}$ is the Bohr magneton and $\mathrm{g}_{0}$ and $\mathrm{g}_{\mathrm{e}}$ - $\mathrm{g}$-factors of a free electron and electron in GaAs and AlAs for $\vec{B} \perp z$. The coefficients $\mathrm{q}_{1}$ and $\mathrm{q}_{2} \mathrm{~g}$-factor of heavy holes; for the symmetry $\mathrm{D}_{2 \mathrm{~d}} \mathrm{q}_{2}=0$.

In the model of Refs. $[2,3]$ the hole wave function of the hh exciton, which includes the hh-lh mixing, has a form:

$\Psi_{ \pm 3 / 2}=\left(| \pm 3 / 2\rangle \mp i \frac{d \varepsilon_{x y}}{\Delta_{l h}}|\mp 1 / 2\rangle\right) C(z)$,

where $\mathrm{d}$ is the shear deformation potential, $\Delta_{\mathrm{lh}}$ is the distance between the lowest $\mathrm{lh}$ and hh levels, $C(z)=$ $C(-z)$ a symmetric function with the origin $z=0$ in the center of the well. In this model we have

$\delta=-\frac{4}{\sqrt{3}} \frac{d \varepsilon_{x y}}{\Delta_{l h}}\left(\Delta-\frac{1}{2} \Delta_{1}\right)$

$q_{1}=q, \quad q_{2}=\frac{4}{\sqrt{3}} k \frac{d \varepsilon_{x y}}{\Delta_{l h}}$.

Therefore, $\mathrm{q}_{2}$ and $\delta$ are connected by the relation

$q_{2}=-k \frac{\delta}{\Delta-\frac{1}{2} \Delta_{1}}$

Here $k$ and $q$ are Luttinger coefficients for the bulk material, $\Delta=\Delta_{0}+\frac{9}{4} \Delta_{1}$ is half the distance between the states of the hh exciton with momentum $M= \pm 1$ and $M= \pm 2$, and $\Delta_{1}$ is half the splitting of the exciton states with $M= \pm 2$. According to Refs. [2, 3], $\left|d \varepsilon_{x y}\right|=14.5 \pm 1.5 \mathrm{meV}$.

In the second model 4,5 

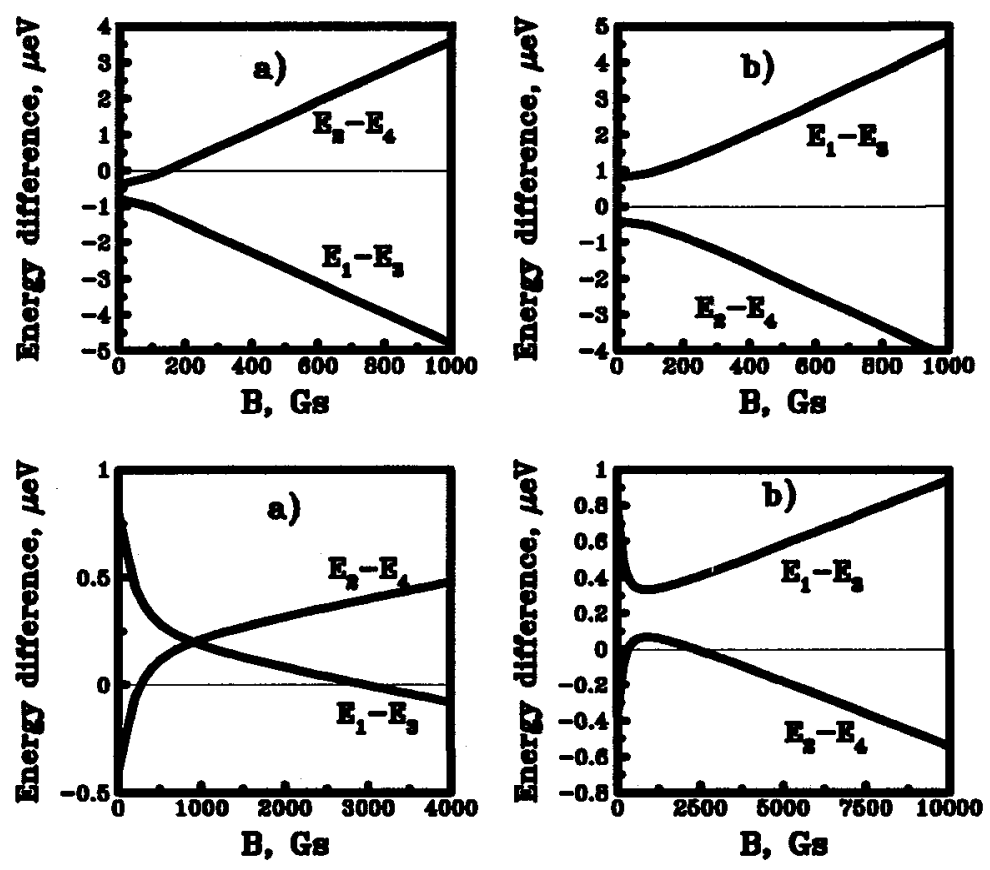

Fig. 1. The dependence of the energy differences $E_{1}$ $E_{3}$ and $E_{2}-E_{4}$ on the magnetic field, model of Refs. [2,3]. The superlattice parameters are $\Delta=-0.82$ $\mu \mathrm{eV},|\delta|=0.4 \mu \mathrm{eV}, \Delta_{1}=0.2$ $\mu \mathrm{eV}, g_{\mathrm{e}}=1.975, q_{1}=-3 \cdot 10^{-2}$, $\left|q_{2}\right|=0.17$. a) $q_{2}>0, \gamma>0$, b) $q<0, \gamma>0$.

Fig. 2. The dependence of the energy differences $E_{1}$ $E_{3}$ and $E_{2}-E_{4}$ on the magnetic field, model of Refs. $[4,5]$. The superlattice parameters are the same as for Fig. 1, but $\left|q_{1}\right|=3 \cdot 10^{-2}$, $q_{2}=0$. a) $q_{1}>0, \gamma \delta>0$, b) $q_{1}<0, \gamma \delta>0$

$\Psi_{ \pm z / 2}=| \pm 3 / 2\rangle C(z) \pm i|\mp 1 / 2\rangle S(z)$,

where $S(z)=-S(-z)$ is an antisymmetric function. In this model

$$
\begin{aligned}
& \delta=\frac{4}{\sqrt{3}}\left(\Delta-\frac{1}{2} \Delta_{1}\right) \frac{\left\langle C(z) S(z)\left|\varphi_{e}(z)\right|^{2}\right\rangle}{\left\langle C(z)^{2}\left|\varphi_{e}(z)\right|^{2}\right\rangle}, \\
& q_{1}=q+q^{\prime}, \quad q^{\prime}=\frac{4}{3} k \frac{\left\langle S(z)^{2}\right\rangle}{\left\langle C(z)^{2}\right\rangle}, \quad q_{2}=0,
\end{aligned}
$$

where $\langle f(z)\rangle=\int_{0}^{a} f(z) d z, a$ is the superlattice period, and $\varphi_{e}(z)$ is an electron wave function. Contrary to Eq. (6), where the correction to the hole g-factor is linear in lh-hh mixing, here the correction is only quadratic, and, therefore, much smaller. The estimation, based on Eq. (6), shows that if $a$ changes from $34 \AA$ to $100 \AA, q_{2}$ is changed from 0.1 to 1 (at the equal thicknesses of the well and the barrier). Thus, for the sample, used in Ref. [1], with $a=45 \AA, \Delta=-0.82 \mu \mathrm{eV},|\delta|=0.4 \mu \mathrm{eV}, \Delta_{1}=0.2 \mu \mathrm{eV}$, and $k=-0.4$, equation (6) gives $\left|q_{2}\right|=0.17$, while the experimental results suggest that $q<10^{-2}$.

In the second model, according to Ref. [4], the ratio $\left\langle S(z)^{2}\right\rangle /\left\langle C(z)^{2}\right\rangle$ can be approximated by the formula $7 \cdot 10^{-3} \exp \left(-0.01 a\right.$ ) ( $a$ in $\AA$ ), so, from Eq. (10) we get $q_{1}$ within the interval from $-0.27 \cdot 10^{-3}$ to $1.4 \cdot 10^{-3}$, which does not contradict the data of Ref. [1].

An effective method to determine the heavy hole g-factor at small $q$ can be provided by the polarization measurements of the exciton luminescence in a magnetic field, parallel to [110] or [1 $\overline{1} 0]$, and under the excitation, polarized along [100] or [010] (or under the circularly polarized excitation). It was shown in Ref. [6], that in a certain magnetic field $B$ the excitonic states, split at $B=0$, undergo a 
level crossing. As a result, the luminescence of the corresponding states becomes polarized in the vicinity of each level crossing. From the hamiltonian (3), the energies of the four excitonic states for $B \|[110]$ or $B \|[1 \overline{1} 0]$ are

$$
\begin{aligned}
& E_{1,2}=\frac{1}{2}\left(\delta-\gamma \Delta_{1}\right) \pm\left\{\left[-\Delta+\frac{1}{2}\left(\delta+\gamma \Delta_{1}\right)\right]^{2}+\left|\delta_{\mathrm{B}}\right|^{2}(1-\alpha)^{2}\right\}^{1 / 2} \\
& E_{3,4}=-\frac{1}{2}\left(\delta-\gamma \Delta_{1}\right) \pm\left\{\left[-\Delta-\frac{1}{2}\left(\delta+\gamma \Delta_{1}\right)\right]^{2}+\left|\delta_{\mathrm{B}}\right|^{2}(1+\alpha)^{2}\right\}^{1 / 2} .
\end{aligned}
$$

where $\left|\delta_{\mathrm{B}}\right|^{2}=\left(1 / \sqrt{2} g_{e} \mu_{\mathrm{B}} B\right)^{2}, \gamma=2 B_{x} B_{y} / B^{2}$, and $\alpha=3 g_{0} / 2 g_{e}\left(\gamma q_{1}+q_{2}\right)$. From Eq. (11) one can see that in the model of Refs. [4, 5], where $q_{2}=0$, the level crossing exists for any sign of $\gamma$ and $q_{1}$ : namely, the terms $E_{1}$ and $E_{3}$ cross, if $\gamma \delta q_{1}>0$, and the terms $E_{2}$ and $E_{4}$ cross, if $\Delta_{1} q_{1}>0$. In the model of Refs. [2, 3], where $q_{2} \gg q_{1}$ and $q_{2} \delta<0$, the terms $E_{2}$ and $E_{4}$ have a crossing, if $\gamma \Delta_{1} q_{2}>0$, and the terms $E_{1}$ and $E_{3}$ do not cross (or have an even number of crossings). Figs. 1 and 2 show the dependence of $E_{1}-E_{3}$ and

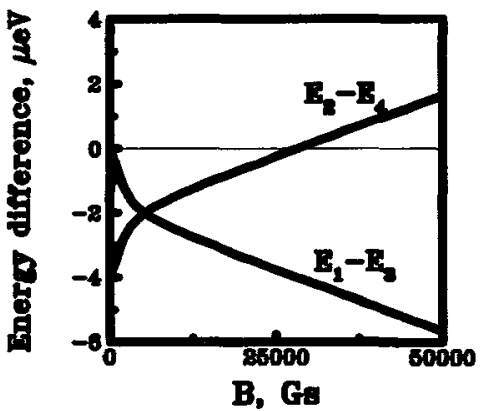

Fig. 3. The dependence of the energy differences $E_{1}-E_{3}$ and $E_{2}-E_{4}$ on the magnetic field for a type I superlattice. The superlattice parameters are $\Delta=-8.2 \mu \mathrm{eV}, \delta=0$, $\Delta_{1}=2 \mu \mathrm{eV}, g_{\mathrm{e}}=1.975$, $q_{1}=3 \cdot 10^{-2}, q_{2}=0, \gamma=1$.
$E_{2}-E_{4}$ on $B$ in the two models.

Note, that the similar level crossing should occur in type I superlattices, where $\delta=0$ and $q_{2}$ $=0$. In this case the luminescence under the excitation, described above, is completely polarized at $B=0$. With increasing magnetic field the polarization disappears, and then reappears again at

certain values of $B$. These phenomena are illustrated on Fig. 3 .

In conclusion, we have calculated the effect of the magnetic field on the exciton levels splitting in GaAs/AlAs type II superlattices. The exciton luminescence, while completely non-polarized at zero magnetic field, becomes partially polarized along the directions [110] or [1 10$]$ at some specific values of the magnetic field. At these points, a level crossing occurs and one of the splittings in the exciton multiplet becomes zero. The corresponding luminescence line will be polarized, while the other exciton lines will remain non-polarized. The values of the magnetic field, at which the level crossings occur, can be found from Figs. 1-3. We show that these values are very different in the two models of the additional exchange splitting. Thus, by performing the measurements described, one can one can assert the validity of one of the models.

We are grateful to E L. Ivchenko and P. Lavallard for helpful discussions. G. E. P. acknowledges the financial support by A. Soros foundation, awarded by American Physical Society.

[1] H. W. van Kesteren, E. C. Cosman, F. J. A. M. Greindanus, P. Dawson, K. J. Moore, C. T. Foxon, Phys. Rev. Lett., 61 (1988), 129.

[2] C. Gourdon, P. Lavallard, Phys. Rev. B, 46 (1992), 4644.

[3] P. Lavallard, C. Gourdon, Superlatt. and Microstructruct., 12 (1992), 321.

[4] I. L. Aleiner, E. L. Ivchenko, Pis'ma Zh. Exper. Teor. Fiz., 55 (1992), 662 [English transl. JETP Letters, 55 (1992), 692.]

[5] E. L. Ivchenko, A. Yu. Kaminskii, I. L. Aleiner, Zh. Exper. Teor. Fiz., (1993), in press.

[6] G. E. Pikus, F. G. Pikus, J. of Lumin., 54 (1993), 279. 